\title{
Validation of an equation for estimating maximal oxygen consumption of nonexpert adult swimmers
}

\section{Adalberto Veronese da Costa ${ }^{1,2}$ Manoel da Cunha Costa ${ }^{3}$ Saulo Fernandes Melo de Oliveira $^{3}$ \\ Fabíola Lima de \\ Albuquerque $^{3}$ Fernando José de Sá Pereira Guimarães $^{3}$} Tiago Manuel Barbosa ${ }^{4}$

'Department of Physical Education, Bioscience Laboratory of Human Kinetics, Rio Grande do Norte State University, Mossoró, Brazil; ${ }^{2}$ Sport Sciences Trás-os-Montes e Alto Douro University, Research Center in Sport, Health and Human Development, Vila Real, Portugal; ${ }^{3}$ Superior School of Physical Education, Human Performance Laboratory, Pernambuco State University, Recife, Brazil; ${ }^{4}$ National Institute of Education, Nanyang Technological University, Singapore
Correspondence: Adalberto Veronese da Costa

Physical Education College, BR

I I0 - Km 48 (Campus-UERN),

Costa e Silva Area, 59610-090

Mossoró - RN, Brazil

Tel +55843315 2131

Fax +55843315 213।

Email adalberto.ef@gmail.com
This article was published in the following Dove Press journal:

Open Access Journal of Sports Medicine

18 January 2013

Number of times this article has been viewed

Objective: To validate an equation to estimate the maximal oxygen consumption $\left(\mathrm{VO}_{2} \max \right)$ of nonexpert adult swimmers.

Methods: Participants were 22 nonexpert swimmers, male, aged between 18 and 30 years (age: $23.1 \pm 3: 59$ years; body mass: $73.6 \pm 7: 39 \mathrm{~kg}$; height $176.6 \pm 5.53 \mathrm{~cm}$; and body fat percentage: $15.9 \% \pm 4.39 \%$ ), divided into two subgroups: $\mathrm{G} 1$ - eleven swimmers for the $\mathrm{VO}_{2}$ max oximetry and modeling of the equation; and G2 - eleven swimmers for application of the equation modeled on G1 and verification of their validation. The test used was the adapted Progressive Swim Test, in which there occurs an increase in the intensity of the swim every two laps. For normality and homogeneity of data, Shapiro-Wilk and Levene tests were used, the descriptive values of the average and standard deviation. The statistical steps were: (1) reliability of the Progressive Swim Test - through the paired $t$-test, intraclass correlation coefficient (ICC), and the Pearson linear correlation $(\mathrm{R})$ relative to the reproducibility, the coefficient of variation $(\mathrm{CV})$, and standard error measurement (SEM) for the absolute reproducibility; (2) in the model equation to estimate $\mathrm{VO}_{2}$ max, a relative $\mathrm{VO}_{2}$ was established, and a stepwise multiple regression model was performed with $\mathrm{G} 1$ - so the variables used were analysis of variance regression (AR), coefficient of determination $\left(\mathrm{R}^{2}\right)$, adjusted coefficient of determination $\left(\mathrm{R}^{2} \mathrm{a}\right)$, standard error of estimate (SEE), and Durbin-Watson (DW); (3) validation of the equation - the results were presented in graphs, where direct (G1) and estimated (G2) $\mathrm{VO}_{2}$ max were compared using independent $t$-test, linear regression (stressing the correlation between groups), and Bland-Altman (the bias agreement of the results). All considered a statistical significance level of $P<0.05$.

Results: On the trustworthiness of the Progressive Swim Test adapted presented as high as observed ( $\mathrm{R}$ and $\mathrm{ICC}>0.80, \mathrm{CV}<10 \%$, and $\mathrm{SEM}<2 \%$ ). In the equation model, $\mathrm{VO}_{2} \max$ has been considered the third model as recommended due to the values found ( $\mathrm{AR}<0.01$, $\mathrm{R}=0795, \mathrm{R}^{2}=0633 ; \mathrm{R}^{2} \mathrm{a}=0.624, \mathrm{SEE}=7.21, \mathrm{DW}=2.06$ ). Upon validation of the equation, no significant differences occurred between G1 and G2 $(P>0.01)$, linear regression stressed a correlation between the groups ( $\mathrm{R}>0.80, P<0.01$ ), and Bland-Altman plotting of the results was within the correlation limits of 1.96 ( $95 \%$ confidence interval).

Conclusion: The estimating equation for $\mathrm{VO}_{2}$ max for nonexpert swimmers is valid for its application through the Progressive Swim Test, providing to contribute in prescribing the swimming lessons as a method of evaluating the physical condition of its practitioners.

Keywords: swimming, $\mathrm{VO}_{2} \max$, regression equation, health

\section{Introduction}

The evaluation of the maximum oxygen consumption $\left(\mathrm{VO}_{2} \max \right)$ has been used as a parameter to determine the intensity of physical activity during aerobic exercises. ${ }^{1,2}$ In swimming, the direct test that presents the conditions to analyze the $\mathrm{VO}_{2}$ max is the 
aquatic ergospirometry, ${ }^{3}$ which enables quantification of the respiratory capacity due to the swimmer's ability to maintain a maximum rate of muscle work during swimming., ${ }^{4,5}$ Therefore, some direct methods have been used to assess the distance traveled during swimming; Castro et al ${ }^{6}$ highlights the swim flume, tethered swimming, back extrapolation from recovery, and with collection during swimming, as the methods most frequently used to evaluate the $\mathrm{VO}_{2} \max$. With this technology, assessments have been performed at the pool approximating them to the sport reality, as the conditions of real life can substantially influence the answers of the cardio respiratory exercise, becoming the direct methods of assessment in the aquatic environment ideals for a better accuracy in swimming. ${ }^{7-9}$

The assessments performed by direct measurement of oxygen consumption present disadvantages and advantages to be considered. The disadvantages are at a high financial cost due to the sophistication of the equipment and some limitations in the biomechanical aspect (ie, absence of side breathing and the, impossibility of running the Olympic turn, for front crawl, as well as the drag of the equipment used where the swimmer performs the swim with a valve which captures the air through the system breath-by-breath). Its advantages are the possibility of the direct analysis to validate new assessment tools in the aquatic environment through the respiratory exchange required in the tests. ${ }^{10}$ With this perspective, several measures have been taken to verify the effectiveness of new equipment for oximetry, ${ }^{11-13}$ analysis of energy expenditure in competitive swimming, ${ }^{2}$ comparing the performance of elite swimmers with nonexpert swimmers, ${ }^{14}$ and to validate the prediction equations. The prediction equations resulting from the direct test results have contributed as a valuation method of low cost and applicability. With swimming, it is possible to identify these equations from the anthropometric viewpoint, ${ }^{15}$ estimating the propulsive force of swimming, ${ }^{16}$ at the critical speed, ${ }^{17}$ as well as validating tests to determine the aerobic capacity. ${ }^{18}$ Therefore, the determination of an equation is linked to the type of test to be performed. In this case, the tests that are fit to be applied in nonexpert swimmers are those that enable the intensity of swimming to be controlled. It is believed that the progressive tests would be ideal for assessing the $\mathrm{VO}_{2} \max$ due to controlling the rate of stroke without intervals, which would gradually increase the intensity and duration through sounds and signals, mainly because these swimmers do not have the expertise to properly control their rhythm during swimming, as do elite swimmers. ${ }^{19,20}$ It is also worth mentioning that the execution time of swimming at a speed that matches the maximal oxygen uptake for nonexpert swimmers is between 310 and 325 seconds, ${ }^{21,22}$ which requires attention to the duration of test execution for these swimmers.

When dealing with the studies which involve nonexpert swimmers, verified results focus on propulsive efficiency of swimming and the changes that occur at different levels of performance for certain distances, as it is common to consider that when a particular subject is experiencing changes in mechanical efficiency this factor is being related to the efficiency of motion due to the process of physical training and thus enables swimming a progressively greater distance..$^{23,24}$ In this regard, the intensity of the activities proposed in swimming lessons needs further discussion, especially when it comes to people who practice regular noncompetitive physical activity, since the number of studies that evaluate the performance in elite swimming athletes is greater than those for nonexpert swimmers, and it seems that there is no equation for determining the level of $\mathrm{VO}_{2}$ max for swimmers of this level. 19,23,24 $^{2}$

Therefore, this study aimed to validate an equation for estimating the $\mathrm{VO}_{2} \max$ of nonexpert adult swimmers. The hypothesis is that there is a high correlation between the direct $\mathrm{VO}_{2}$ max and the equation for estimating $\mathrm{VO}_{2}$ max.

\section{Methodology Subjects}

The study included 22 nonexpert male swimmers, aged between 18 and 30 years (age: 23.1 $\pm 3: 59$ years; body mass: $73.6 \pm 7: 39 \mathrm{~kg}$; height $176.6 \pm 5.53 \mathrm{~cm}$; and body fat percentage: $15.9 \% \pm 4.39 \%$ ). These swimmers were divided into two subgroups: G1, in which eleven swimmers were evaluated through direct $\mathrm{VO}_{2}$ max for modeling the equation; and $\mathrm{G} 2$, in which eleven swimmers were used for application of the equation modeled on G1, aiming to verify its validation.

The following inclusion criteria were defined: swimmers with accurate technique for front crawl; they practice swimming lessons three times a week with a duration of 1 hour per session, swimming about 800 meters; they have not taken any kind of dietary supplementation; and have not done any physical activity 24 hours before the test. The following exclusion criteria were applied: elite swimmers affiliated with the national federation for swimming or athletes who had been affiliated 3 years before the survey; nonexpert swimmers who had responded negatively to one of the questions in the Readiness Questionnaire for Practice Physical Activity; ${ }^{25}$ had executed the test in under 4 minutes; $;^{20,22,26}$ or visibly presented 
any kind of illness that would limit the study (eg, flu, fever, or any type of injury).

Regarding the experimental design in dealing with quantitative nonexpert swimmers, despite swimming schools that agreed to participate in the survey presented a number of people who were in the learning phase of the swimming strokes or of nonexpert swimmers who were outside the required age, the quantitative subject to the completion of this study has become feasible in accordance with other studies in the area. ${ }^{14,28-30}$

\section{Data collection}

The test used for the validation of the equation for estimating $\mathrm{VO}_{2} \max$ was the Progressive Swim Test proposed by Veronese da Costa et al. ${ }^{19}$ This protocol corresponds to a progressive series of front crawl in a $25 \mathrm{~m}$ pool and includes the use of a recording of beeps that enable the swimmer to keep the correct swimming rhythm; there is a decrease of the partial times of 1 second for each lap completed (number of laps performed [NLP]). The test ends when the swimmer twice followed fails to be within 5 meters that precede the edge of the pool. Studies have shown that $\mathrm{VO}_{2} \max$ in a swimming test is obtained above the 4 minutes to meet the criteria for verification of $\mathrm{VO}_{2} \max$ (an increase of $45 \mathrm{~mL} /$ $\mathrm{kg} / \mathrm{min}$; rate of perceived exertion of seven, classified as "very hard;" 90\% maximal heart rate). ${ }^{6,27}$ The protocol of Veronese da Costa et al was adapted to extend the time of exercise and verification of all the criteria for reaching $\mathrm{VO}_{2}$ max. ${ }^{6,19,20,26}$ Accordingly, the adaptation has doubled the maximum distance from 400 to $800 \mathrm{~m}$, with the goal of increasing the intensity of the test with the reduction of 1 second for every two laps completed. In the first two laps of $25 \mathrm{~m}$, the swimmer executed the test with the proposed time of $28^{\prime \prime} 30(0.88 \mathrm{~m} / \mathrm{s}$ or $18.3 \mathrm{~km} / \mathrm{h})$ and a total time until the second round of 56"36; the next two laps were performed with a time of $27^{\prime \prime} 30(0.92 \mathrm{~m} / \mathrm{s}$ or $30.3 \mathrm{~km} / \mathrm{h})$ and a total time up to the fourth round of $01^{\prime} 51^{\prime \prime} 00$, and so on. It was observed that the minimum time was about four minutes between the ninth and the tenth lap (time $24 " 30,1.3 \mathrm{~m} / \mathrm{s}$ or $3.70 \mathrm{~km} / \mathrm{h}$ ), confirming the criteria used in this study. Heart rate was checked before the test to identify the swimmer's resting conditions, so it was used as criteria to start the test (heart rate below $90 \mathrm{bpm}$ ), and after the test (AHR) to verify that the maximum heart rate reached. During the test, the swimmer used an underwater MP3 device which was attached to the silicon strip of the swimmer's goggles (SwiMP3 V2, Finis Inc, Livermore, CA), and the evaluators had a microsystem of 50-60 Hz (18 W). Thus, both the swimmer and the evaluators followed the beeps during the test. The oxygen consumption, as well as other respiratory and metabolic variables, was assessed by a metabolic card (K4b2, Cosmed ${ }^{\circledR}$, Rome, Italy) being connected to a snorkel (Aquatrainer $^{\circledR}$, Cosmed $^{\circledR}$, Rome, Italy) fixed in the mouth of the swimmer.9,28-30 To control the cardiovascular response, a heart rate monitor (Polar FT1 Model, Polar Electro Oy, Kempele, Finland) was used. To analyze the perceived exertion, Borg's category-ratio scale was used at the end of the protocol. ${ }^{27,31}$

\section{Ethical aspects}

All the procedures followed the guidelines of the Declaration of Helsinki in dealing with the ethics involving human subjects. This study was approved by the Ethics Committee of the University of Pernambuco/PROPEGE/UPE, Brazil Protocol 035/2012. Before the tests, all participants signed a Free and Clarified Consent.

\section{Statistical analysis}

The sample size in relation to the local swimming schools was estimated using $\mathrm{G}^{*}$ Power software, version 3.0.10 (Franz Faul, University of Kiel, Kiel, Germany). ${ }^{32}$ Thus, by examining the high correlation between $\mathrm{VO}_{2}$ and the distance swum, an ES of 0.75 was observed. Thus, considering a large power of the analyses starting from 0.8 and a corresponding alpha error of 0.05 , the minimum sample of eleven swimmers was reached for this investigation, and it was used for the 22 swimmers' test performances which made up the two subgroups, G1 $(\mathrm{n}=11)$ and G2 $(\mathrm{n}=11)$. For normality and homogeneity of data, the tests of Shapiro-Wilk and Levene and the descriptive values through the mean and standard deviation were used. The following statistical steps were performed.

\section{Reliability of the Progressive Swim Test}

To assess the reliability of NLP, as the adjustment proposed in the Progressive Swim Test, G2 performed the test twice with an interval of 1 week. The paired $t$-test, the relative reproducibility through the intra class correlation coefficient (ICC) and of the linear correlation coefficient (R), as well as for absolute reproducibility, coefficient of variation (CV), and standard error measurement (SEM), as proposed by Veronese da Costa et al, ${ }^{19}$ were used as the criteria, considering that the higher the ICC and R, and the lower the percentage of CV and SEM, the greater will be the levels of reliability testing. 


\section{Model of the $\mathrm{VO}_{2}$ max equation}

for nonexpert adult swimmers

To establish the prediction equation, a stepwise multiple regression model was conducted with $\mathrm{G} 1$ based on the verification of the $\mathrm{VO}_{2}$ of all NLP in progression performed by the swimmers. Thus, there were three combinations of one to three variables (equation I, BM; equation II, NLP and BM; equation III, BM, NLP, and AHR) being selected to be those which showed more influence on the test results. ${ }^{19}$ Analysis of variance regression (AR) was applied to determine possible differences between the variables; in this case, the coefficient of determination $\left(\mathrm{R}^{2}\right)$ and adjusted coefficient of determination $\left(R^{2} a\right)$ served to check the power of the equation proposed for use in $\mathrm{G} 2$, values which were classified as very weak if $\mathrm{R}^{2}<0.04$, weak if $0.04 \leq \mathrm{R}^{2}<0.16$, moderate if $0.16 \leq \mathrm{R}^{2}<0.49$, high if $0.49 \leq \mathrm{R}^{2}<0.81$, and very high if $0.81 \leq \mathrm{R}^{2}<1.0 .^{15}$ The standard error of estimate (SEE) was also used to verify the accuracy of the sample mean calculated, and the Durbin-Watson (DW) test was used to verify the presence of any residue between the variables used in the equation (BM, NLP, AHR).

\section{Validation of the equation}

Following the proposal of Morais et a ${ }^{15}$ for validation of the proposed equation, first, the results of the direct $\mathrm{VO}_{2} \max$ (G1) and the estimated $\mathrm{VO}_{2} \max$ (G2) were compared using an independent $t$-test to check the significance of the results. A simple linear regression model was then applied to identify the level of correlation between groups, and finally, the BlandAltman plot was used to check the bias and limits of agreement between the results: compliance limit $=(1.96 \times$ standard deviation) \pm mean differences. The entire statistical treatment considered a significance level of $P<0.05$.

\section{Results}

\section{Reliability of the Progressive Swim Test}

Table 1 shows the descriptive data, comparative and correlational, of the maximum number of laps completed by nonexpert swimmers in the Progressive Swim Test.
In comparing the data, it was observed that there were no significant differences between test days, the reproducibility was obtained on one R, with ICC exceeding 0.80 (classified as very high absolute reproducibility), a CV under $10 \%$, and an SEM under $2 \%$ of magnitude, confirming a high reliability of the Progressive Swim Test for this distance.

\section{Model of the equation of $\mathrm{VO}_{2} \max$ for nonexpert adult swimmers}

Table 2 highlights three regression models of $\mathrm{VO}_{2} \max$ through each lap completed by the nonexpert swimmers as the progression required by the test. The BM, NLP, and AHR were therefore part of the equation, and taking the observed variables from the regression analysis into consideration, the third model stood out as the most recommended, by presenting results in a higher power compared with the others.

\section{Validation of the equation}

Figure 1 shows the comparison between the average values, linear regression, and Bland-Altman between $\mathrm{VO}_{2}$ max and $\mathrm{VO}_{2}$ directly estimated from model 3 of the equation. The results showed no significant differences between direct VO2max $(44.2 \pm 5.36)$ and $\mathrm{VO}_{2} \max (46.6 \pm 8.67)$ in their mean values $(P>0.05)$. With linear regression, it was observed that there was a correlation between the two groups ( $\mathrm{R}=0.817, P<0.01$ ); with Bland-Altman, no results were found beyond the concordance limit of 1.96 .

\section{Discussion}

The data obtained showed that the equation for estimating $\mathrm{VO}_{2} \max$ performed through the Progressive Swim Test showed evidence of a high power to its applicability, which will be a useful instrument for the implementation of swimming programs of inherent individuality of each swimmer.

\section{Regarding the trustworthiness of the Progressive Swim Test to $800 \mathrm{~m}$}

The adaptation of the Progressive Swim Test was made with the perspective of bringing the swimmer to exhaustion

Table I Statistical analyses of the reliability of the $800 \mathrm{~m}$ Progressive Swim Test

\begin{tabular}{|c|c|c|c|c|c|c|}
\hline \multicolumn{2}{|l|}{ Variables } & \multicolumn{5}{|c|}{$\begin{array}{l}\text { Analysis between NPL day I (NPLI) and } \\
\text { NPL day } 2 \text { (NPL2) }\end{array}$} \\
\hline $\begin{array}{l}\text { NPLI } \\
(\text { mean } \pm \text { SD) }\end{array}$ & $\begin{array}{l}\text { NPL2 } \\
(\text { mean } \pm \text { SD) }\end{array}$ & $\mathbf{R}$ & SEM & $\begin{array}{l}\text { ICC } \\
(95 \% \mathrm{Cl})\end{array}$ & $t$-test & CV \\
\hline $12.3 \pm 4.73$ & $12.4 \pm 4.76$ & $0.974^{a}$ & 0.28459 & $0.986^{a}(0.947 / 0.996)$ & $-0.319^{a}$ & $5.1 \%$ \\
\hline
\end{tabular}

Note: ap $<0.05$.

Abbreviations: NLP, number of laps performed; SD, standard deviation; R, Pearson correlations between NLPI and NPL2; SEM, standard error measurement; ICC, intraclass coefficient correlations; CV, coefficient of variations; Cl, confidence interval. 
Table 2 Models of equation for determination of $\mathrm{VO}_{2}$ max for nonexpert adult swimmers

\begin{tabular}{llllllll}
\hline Model & Regression equations & $\mathbf{A R}^{\mathbf{a}}$ & $\mathbf{R}$ & $\mathbf{R}^{\mathbf{2}}$ & $\mathbf{R}^{\mathbf{a}}$ & $\mathbf{S E E}$ & $\mathbf{D W}$ \\
\hline I & $13.672+2.338(\mathrm{NLP})$ & $<0.01$ & 0.742 & $0.55 \mathrm{I}$ & 0.548 & 7.9127 & 1.643 \\
II & $25.797+2.395(\mathrm{NLP})-0.163(\mathrm{BM})$ & $<0.01$ & 0.769 & 0.592 & 0.586 & 7.5734 & 1.813 \\
III & I4.085 + I.858 (NLP) $-0.192(\mathrm{BM})+0.1 \mathrm{II}(\mathrm{AHR})$ & $<0.01$ & 0.795 & 0.633 & 0.624 & 7.2140 & 2.065 \\
\hline
\end{tabular}

Note: ${ }^{a} P<0.05$.

Abbreviations: $\mathrm{VO}_{2}$ max, maximal oxygen consumption; NLP, number of laps performed; BM, body mass (in kg); AHR, after heart rate test (in beats per minute); AR, analysis of variance regression; R, Pearson correlation coefficient; $R^{2}$, coefficient of determination; $R^{2} a$, adjusted coefficient of determination; SEE, standard error of estimate; DW, Durbin-Watson test.

by increasing the intensity and was determined by the time required for each pool and NLP. In G2 the AHR was over $180 \mathrm{bpm}$ and the RPE over seven, the average time on different days was 5 minutes, and the average speed for the twelfth full lap was $1.07 \mathrm{~m} / \mathrm{s}$ or $3.86 \mathrm{~km} / \mathrm{h}$ (Table 1 ), which showed that nonexpert swimmers performed the test at full performance. Thus, Montpetit et $\mathrm{al}^{20}$ and Castro et $\mathrm{al}^{6}$ emphasize that tests in a swimming pool that enable conditions for the determination of $\mathrm{VO}_{2}$ max to be continuously

\section{A}

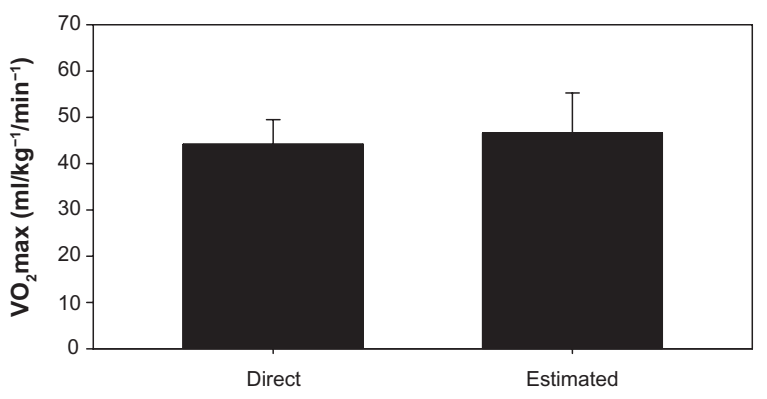

B
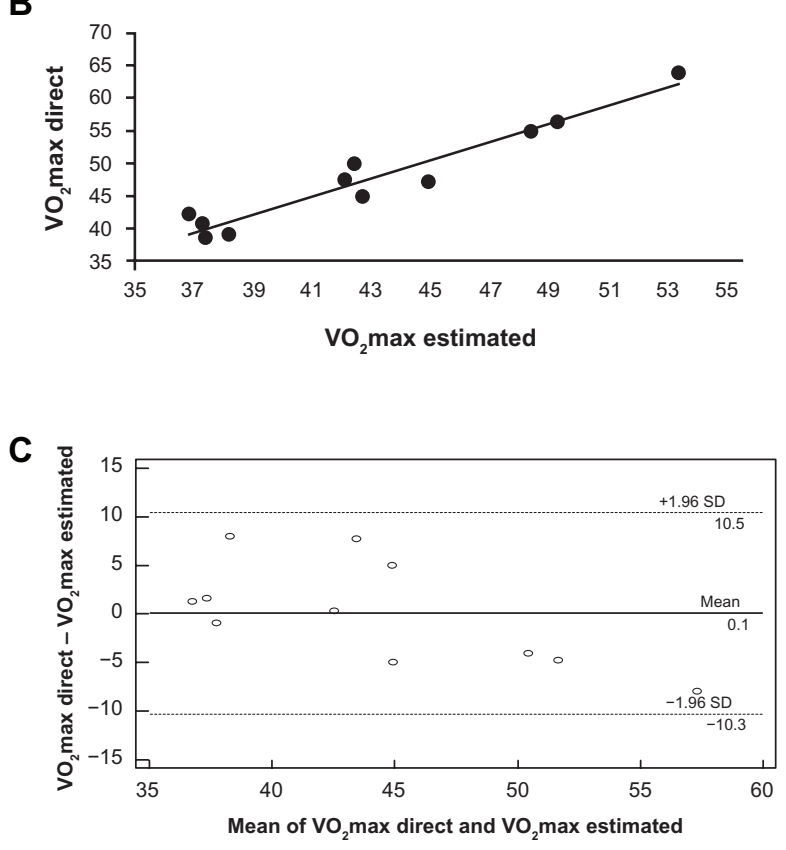

Figure I Comparison of average data, scatter plots, and Bland-Altman between direct and estimated $\mathrm{VO}_{2}$ max of nonexpert adult swimmers. progressive, in intensity does not supra-maximum, that lead the swimmer to exhaustion and lasting longer than 4 minutes. The Progressive Swim Test, required by these performance criteria for nonexpert swimmers, allows its application for the determination of $\mathrm{VO}_{2} \max$ from a validation through direct measurement of oxygen consumption. ${ }^{19}$

By noting that the values of NLP between different days showed no significant differences $(P>0.05)$, the values of trustworthiness made the following classifications: very high (R $>0.80, P<0.05$ ), excellent agreement (ICC $>0.90$, $P<0.05)$, SEM $(95 \% \mathrm{CI}<2 \%)$, and $\mathrm{CV}<10 \%$; when $\mathrm{R}$ and ICC are high and SEM and CV are below their required limits, the test has proven trustworthiness for application on nonexpert swimmers.

\section{Regarding the proposed equation}

To the creation of the equation for estimating the $\mathrm{VO}_{2} \max$ for nonexpert swimmers to relativize the $\mathrm{VO}_{2} \max$ it was needed by the $\mathrm{BM}\left(\mathrm{VO}_{2} \max \left[\mathrm{ml} / \mathrm{kg}^{-1} / \mathrm{min}^{-1}\right]=\mathrm{VO}_{2} \max \right.$ $\left[\mathrm{ml} / \mathrm{min}^{-1}\right]$ X BM $[\mathrm{kg}]$ ) on each lap completed by the nonexpert swimmers, allowing you to check in how many pools swimmer can swim. To Smith et $\mathrm{al}^{22}$ and Montpetit, Smith, and Boie, ${ }^{33}$ this relativization is satisfactory from the point of view of cancellation of the differences between individuals. In this sense, the relative $\mathrm{VO}_{2}$ enabled the creation of three models using BM, NLP, and AHR. These variables were selected because they are the determinants of the physiological conditions of the swimmers in the test. ${ }^{19,34}$

The third model was applied to another group of nonexpert swimmers (G2) with the aim of validation. The differences in AR $(P<0.01)$ statistically prove the existence of regression, and these differences naturally occur as a result in the number of lap performed of the Progressive Swim Test have been developed to increase the swimming intensity every two laps. An R of 0.795 showed that there was a good correlation between swimmers and NLP. As for $\mathrm{R}^{2}$ and $\mathrm{R}^{2} \mathrm{a}$, changes were satisfactory (high if $0.49 \leq \mathrm{R}^{2}<0.81$ ); the ESS of 7\% is considered very low for an indirect test, and the DW independence waste observed showed that the variables 
BM, NLP, and AHR were not interdependent, confirming a high power of the equation.

\section{Regarding the validation of the prediction equation through the Progressive Swim Test}

To validate this equation, it is worth considering the nonexpert swimmers who participated as gold standard presented similar characteristics compared with the group with the results estimated using the equation, but also it should be noted that the equation will be valid for males and age range of this proposal study. When comparing the results of the direct $\mathrm{VO}_{2} \max (\mathrm{G} 1)$ with $\mathrm{VO}_{2} \max$ estimated by the equation (G2), and verifying that there were no significant differences between the groups, it was observed that the average value was within $45 \mathrm{~mL} / \mathrm{kg} / \mathrm{min}$, a result that fits the test performed by Ribeiro et al, which evaluates the end of the $400 \mathrm{~m}$ crawl made with maximum speed and notes that the athlete swimmers presented results between 45 and $62 \mathrm{~mL} / \mathrm{kg} / \mathrm{min} .{ }^{6,34}$ Using linear regression, it was found that there was a correlation between the gold standard and the estimated method, and none of the values were outside the level of the magnitude in the Bland-Altman plot.

The limitations of this study that warrant future studies include: (1) hydrostatic weighing, which would enable the influence of lift of the body in the water to be determined, was not performed; (2) biomechanical analysis of swimming, which would identify efficiency swum through the index of strokes for each lap completed, was not performed; (3) this study should be conducted using subjects in other age groups and levels of swimming, ie, age under 18 years and over 30 years, and elite athletes.

In conclusion, the equation for estimating $\mathrm{VO}_{2} \max$ in nonexpert swimmers is recommended for its application through the Progressive Swim Test, and provides help in prescribing the swimming lessons as a method of evaluating the fitness of its practitioners.

\section{Disclosure}

The authors declare no conflicts of interest in this work.

\section{References}

1. Raffaelli C, Galvani C, Lanza M, Zamparo P. Different methods for monitoring intensity during water-based aerobic exercises. Eur J Appl Physiol. 2012;112(1):125-134.

2. Barbosa TM, Fernandes R, Keskinen KL, et al. Evaluation of the energy expenditure in competitive swimming strokes. Int J Sports Med. 2006;27(11):894-899.

3. Castro FAS, Mota CB. Energetic and performance in $200 \mathrm{~m}$ front crawl under maximal intensity. R bras Ci e Mov. 2010;18(2):67-75.
4. Papoti M, Martins LE, Cunha SA, Zagatto AM, Gobatto CA. Effects of taper on swimming force and swimmer performance after an experimental ten-week training program. J Strength Cond Res. 2007;21(2):538-542.

5. Toussaint H, Meulemans A, de Groot G, Hollander A, Schreurs A, Vervoorn K. Respiratory valve for oxygen uptake measurements during swimming. Eur J Appl Physiol Occup Physiol. 1987;56(3):363-366.

6. Castro FAS, Franken M, Silveira RP, Mota CB. Oxygen consumption in swimming: different methodologies and application possibilities. R bras Ci e Mov. 2010;18(3):88-93.

7. Schnitzler C, Heck G, Chatard J-C, Ernwein V. A simple field test to assess endurance in inexperienced runners. $J$ Strength Cond Res. 2010;24(8):2026-2031.

8. Gayda M, Bosquet L, Juneau M, Guiraud T, Lambert J, Nigam A. Comparison of gas exchange data using the Aquatrainer system and the facemask with Cosmed K4b2 during exercise in healthy subjects. Eur J Appl Physiol. 2010;109(2):191-199.

9. Keskinen KL, Rodriguez FA, Keskinen OP. Respiratory snorkel and valve system for breath-by-breath gas analysis in swimming. Scand $J$ Med Sci Sports. 2003;13(5):322-329.

10. Ferreira IF, Silva AJ, Oliveira D, et al. Analysis of the determinant kinematical parameters for performance in the 200-m freestyle swimming event. Motriz Rev Educ Fis. 2012;18:366-377.

11. Duffield R, Dawson B, Pinnington HC, Wong P. Accuracy and reliability of a Cosmed K4b2 portable gas analysis system. J Sci Med Sport. 2004;7(1):11-22.

12. McLaughlin JE, King GA, Howley ET, Bassett DR Jr, Ainsworth BE. Validation of the COSMED K4 b2 portable metabolic system. Int $J$ Sports Med. 2001;22(04):280-284.

13. Papoti M, Balikian P Jr, Denadai BS, et al. Adaptation of the VO2000 gas analiser's mask to mensuration of cardiorespiratory parameters in swimming. Rev Bras Med Esporte. 2007;13:190-194.

14. Schnitzler C, Seifert L, Chollet D. Arm coordination and performance level in the 400-m front crawl. Res Q Exerc Sport. 2011;82(1):1-8.

15. Morais JE, Costa MJ, Mejias EJ, Marinho DA, Silva AJ, Barbosa TM. Morphometric study for estimation and validation of trunk transverse surface area to assess human drag force on water. J Hum Kinet. 2011;28(1):5-13.

16. Santos MA, Barbosa ML Jr, Melo WV, Veronese da Costa A, Costa MC. Estimate of propulsive force in front crawl swimming in young athletes. Open Access J Sports Med. 2012;3(1):115-120.

17. Franken M, Zacca R, Castro FAS. Critical speed in swimming: theoretical basis and application. Motriz Rev Educ Fiz. 2011;17:209-222.

18. Deminice R, Papoti M, Zagatto AM, Prado Júnior MV. Validity of 30 minutes test (T-30) in aerobic capacity, stroke parameters and aerobic performance determination of trained swimmers. Rev Bras Med Esporte. 2007;13:195-199.

19. Veronese da Costa A, Costa MC, Carlos DM, Guerra LM, Silva AJ, Barbosa TM. Reproducibility of an aerobic endurance test for nonexpert swimmers. J Multidiscip Healthc. 2012;5(1):215-221.

20. Montpetit R, Léger L, Lavoie J-M, Cazorla $\mathrm{G} . \mathrm{VO}_{2}$ peak during free swimming using the backward extrapolation of the $\mathrm{O}_{2}$ recovery curve. Eur J Appl Physiol Occup Physiol. 1981;47(4):385-391.

21. Fernandes RJ, Vilas-Boas $\mathrm{P}$. Time to exhaustion at the $\mathrm{VO}_{2}$ max velocity in swimming: a review. J Hum Kinet. 2012;32(1):121-134.

22. Silva AJ, Machado Reis V, Marinho D, Carneiro AL, Novaes G, Aidar FJ. Swimming economy: determinant factors and assessment issues. Rev Bras Cineantropom Desempenho Hum. 2006;8(3):93-99.

23. Barbosa TM, Lima V, Mejias E, et al. Propulsive efficiency and nonexpert swimmers performance. Motri. 2009;5:27-43.

24. Zamparo P. Effects of age and gender on the propelling efficiency of the arm stroke. Eur J Appl Physiol. 2006;97(1):52-58.

25. Thomas S, Reading J, Shephard RJ. Revision of the Physical Activity Readiness Questionnaire (PAR-Q). Can J Sport Sci. 1992;17(4): 338-345.

26. Fernandes RJ, Vilas-Boas JP. Time to exhaustion at the $\mathrm{VO}_{2}$ max velocity in swimming: a review. J Hum Kinet. 2012;32:121-134. 
27. Wallace LK, Slattery KM, Coutts AJ. The ecological validity and application of the session-RPE method for quantifying training loads in swimming. J Strength Cond Res. 2009;23(1):33-38.

28. Barbosa T, Silva A, Reis A, et al. Kinematical changes in swimming front crawl and breaststroke with the AquaTrainer snorkel. Eur J Appl Physiol. 2010;109(6):1155-1162.

29. Reis $\mathrm{V}$. $\mathrm{VO}_{2}$ underestimation using the Aquatrainer compared with facemask measurements: is it all in the ventilation? Eur J Appl Physiol. 2011;111(6):1223-1224.

30. Robach P, Schmitt L, Brugniaux J, et al. Living high - training low: effect on erythropoiesis and aerobic performance in highly trained swimmers. Eur J Appl Physiol. 2006;96(4):423-433.

31. Borg G, Ljunggren G, Ceci R. The increase of perceived exertion, aches and pain in the legs, heart rate and blood lactate during exercise on a bicycle ergometer. Eur J Appl Physiol Occup Physiol. 1985;54(4):343-349.
32. Faul F, Erdfelder E, Lang A-G, Buchner A. G*Power 3: a flexible statistical power analysis program for the social, behavioral, and biomedical sciences. Behav Res Methods. 2007;39(2):175-191.

33. Montpetit R, Smith H, Boie G. Swimming economy: how to standardize the data to compare swimming proficiency. J Swimming Res. 1988;4(1):5-8.

34. Ribeiro JP, Cadavid E, Baena J, Monsalvete E, Barna A, De Rose EH. Metabolic predictors of middle-distance swimming performance. Brit J Sport Med. 1990;24(3):196-200.

\section{Publish your work in this journal}

Open Access Journal of Sports Medicine is an international, peer-reviewed, open access journal publishing original research, reports, reviews and commentaries on all areas of sports medicine. The manuscript management system is completely online and includes a very quick and fair peer-review system.
Visit http://www.dovepress.com/testimonials.php to read real quotes from published authors. 